\title{
Bi-clustering of Gene Expression Data Using Conditional Entropy
}

\author{
Afolabi Olomola ${ }^{1}$ and Sumeet Dua ${ }^{1,2}$ \\ ${ }^{1}$ Data Mining Research Laboratory (DMRL), Department of Computer Science \\ Louisiana Tech University, Ruston, LA, U.S.A. \\ ${ }^{2}$ School of Medicine, Louisiana State University Health Sciences, New Orleans, LA, U.S.A. \\ $\{$ aol003, sdua\} @latech.edu
}

\begin{abstract}
The inherent sparseness of gene expression data and the rare exhibition of similar expression patterns across a wide range of conditions make traditional clustering techniques unsuitable for gene expression analysis. Biclustering methods currently used to identify correlated gene patterns based on a subset of conditions do not effectively mine constant, coherent, or overlapping biclusters, partially because they perform poorly in the presence of noise. In this paper, we present a new methodology (BiEntropy) that combines information entropy and graph theory techniques to identify co-expressed gene patterns that are relevant to a subset of the sample. Our goal is to discover different types of biclusters in the presence of noise and to demonstrate the superiority of our method over existing methods in terms of discovering functionally enriched biclusters. We demonstrate the effectiveness of our method using both synthetic and real data.
\end{abstract}

Keywords: Gene expression, biclustering, conditional entropy.

\section{Background}

A major challenge in the analysis of gene expression datasets is the discovery of local structures composed of gene sets that show coherent expression patterns across subsets of experimental conditions. These patterns may provide clues about the biological processes associated with physiological states. Recently, researchers have focused on using biclustering methods to find local patterns in which genes in a subset might be similar, based only on a condition subset. Hatigan first defined biclustering as a distinct class of clustering algorithms that perform simultaneous row-column clustering [1]. Cheng and Church first applied biclustering to analyze DNA microarray experimental data [2]. They proposed a greedy algorithm to find a given number of $\delta$ biclusters, whose mean squared residues are less than given thresholds. Kupiec et al. [3] presented SAMBA, a graph-theory approach combined with a statistical data model. In the SAMBA framework, the expression matrix is modeled as a bipartite graph and a likelihood score is used to assess the significance of observed sub graphs. The Preserving Sub-Matrix Algorithm (OPSM) [4] bicluster is defined as a submatrix that preserves the order of the selected columns for all selected rows. Based on 
a stochastic model, the authors developed a deterministic algorithm to find large and statistically significant biclusters.

The Iterative Signature Algorithm (ISA) [5] uses gene signatures and condition signatures to find biclusters with both up- and down-regulated expression values. Murali and Kasif (2003)[6] have proposed xMotif, a framework that seeks biclusters which contain genes that are expressed across the selection of samples. The method involves an iterative search method that runs on random seeds to identify the largest valid biclusters. Zimmerman et al. (2006)[7] have proposed BiMax to find constant biclusters by discretizing the input expression matrix into a binary matrix. However, this discretization makes it harder to determine coherent biclusters.

These biclustering methods are not capable of handling noise and discovering several types of biclusters in gene expression. In this paper, we present a new biclustering method that combines information entropy (conditional entropy) and graph theory techniques to identify co-expression patterns of genes that might be relevant to a subset of the condition. This method is motivated by the use of conditional entropy to measure interdependence between pairs of entities. The measure of interdependence between pairs of conditions and genes helps to predict the presence of a good cluster. Our method transforms conditional entropy between pairs of conditions into an unweighted graph and reduces the need to iteratively search for groups of related conditions to find maximal cliques. This experiment has two objectives: to show that BiEntropy can find constant, coherent, and overlapped bi-clusters even if the cluster contains noise and to demonstrate the superiority of BiEntropy over existing biclustering methods in terms of identifying meaningful gene groups related to Gocategories.

Our bicluster method consists of the following steps: (1) normalization and discretization, (2) generation of conditional matrix, (3) construction of unweighted graph, (4) finding of maximal cliques, and (5) identification of biclusters. The experimental results on both synthetic and real data (Saccharomyces cerevisiae and NCI 60 datasets) demonstrate the effectiveness of BiEntropy in discovering artificially embedded biclusters as well as biologically significant biclusters with high precision.

\section{Definitions and Notations}

In this section, we present a general definition of a bicluster. Let $G=\left\{g_{1}, \ldots \ldots \ldots, g_{M}\right\}$ be a set of genes (rows), and let $C=\left\{c_{1}, \ldots \ldots, c_{N}\right\}$ be a set of conditions (columns). The data can be viewed as an $M \times N$ expression matrix EM, where rows signify genes and columns signify experimental conditions. EM is a matrix of real numbers, where each entry $g_{i j}$ corresponds to the logarithm of the relative abundance of the mRNA of a gene $g_{i}$ under a specific condition $c_{j}$.

A bicluster corresponds to a sub-matrix that exhibits some coherent tendency. Each bicluster can be identified by a unique set of genes and experimental conditions that determine the sub-matrix. Thus, a bicluster is a matrix $I \times J$, denoted as $(I, J)$, where $I$ and $J$ are a set of genes (rows) and conditions (columns), respectively. In this case, $I \subseteq M$, and $J \subseteq N$. We define the volume or size of a bicluster $(I, J)$ as the num- 
ber of elements $g_{i j}$, so that $i \in I$ and $j \in J$. A sub-matrix of $A(I, J)$ with $I \subseteq M$ and $J \subseteq N$ is a constant bicluster for reference gene $g_{i}$ if for any $i \in I$ and any $j \in J, g_{i j}=g_{i}{ }^{*} j_{j}$. A sub-matrix of $A(I, J)$ with $I \subseteq M$ and $J \subseteq N$ is an additive for reference gene $i^{*}$ if for any $i \in I$, and $j \in J, g_{i j}-g_{i}^{*} j=\theta_{i}$, where $\theta_{i}$ is a constant for any row $i$.

\begin{tabular}{|l|l|l|l|}
\hline 1.0 & 1.0 & 1.0 & 1.0 \\
\hline 1.0 & 1.0 & 1.0 & 1.0 \\
\hline 1.0 & 1.0 & 1.0 & 1.0 \\
\hline 1.0 & 1.0 & 1.0 & 1.0 \\
\hline
\end{tabular}

(a) cons tart bichuster

\begin{tabular}{|l|l|l|l|}
\hline 1.0 & 2.0 & 5.0 & 0.0 \\
\hline 2.0 & 3.0 & 6.0 & 1.0 \\
\hline 4.0 & 5.0 & 8.0 & 3.0 \\
\hline 5.0 & 6.0 & 9.0 & 4.0 \\
\hline
\end{tabular}

(b)additive coherent bichster

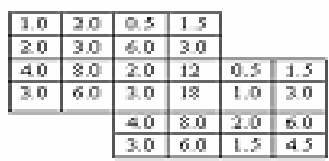

(c) overlapped bichster

Fig. 1. Example of types of biclusters: (a) constant biclusters, (b) coherent (additive), (c) overlapping

\section{Materials and Method}

Before normalizing gene expression data, we temporarily remove data beyond a threshold (three standard deviations) to reduce the effect of outliers. Then, we linearly normalize each condition (column) of data with a mean of 0 and a variance of 1 . We repeat the procedure until no outliers remain. Next, we assign the temporarily removed outlier values equal to the corresponding extreme value of the final normalized data (minimum for outliers below the mean, maximum for outliers above the mean). We discretize each gene expression level into intervals, by uniformly dividing the difference between the maximum and minimum values in the normalized data.

\subsection{Generation of Conditional Entropy Matrix}

We generate a symmetric matrix by finding the conditional entropy between all pairs of conditions (columns) in the discretized data. The conditional entropy measures the mutual interaction between pairs of conditions (column) and predicts each pair's ability to form a good cluster. Higher conditional entropy between a pair of conditions indicates a lower possibility of the entropy forming a significant cluster. Therefore, a low conditional entropy value between two conditions denotes the presence of a clustering relationship between the two conditions.

Lemma 1. Conditional Entropy. Let $\left\{c_{1}^{1}, c_{1}^{2}, c_{1}^{3} \ldots \ldots c_{1}^{K}\right\}$ and $\left\{c_{2}^{1}, c_{2}^{2}, c_{2}^{3} \ldots \ldots c_{2}^{K}\right\}$ be set of intervals in condition (column) $c_{1}$ and $c_{2}$, respectively. We set the conditional entropy for condition $c_{1}$ given $c_{2}$ as:

$$
H\left(c_{1} \mid c_{2}\right)=-\sum_{k=1}^{K} P\left(c_{1}^{k}\right) \sum_{l=1}^{K} P\left(c_{1}^{l} \mid c_{2}^{k}\right) \log P\left(c_{1}^{l} \mid c_{2}^{k}\right) .
$$


$P\left(c_{1}^{k}\right)$ is the probability of data at interval $k$ of sample $c_{1}$, and $P\left(c_{1}^{l} \mid C_{2}^{k}\right)$ is the conditional probability of a data point in the interval $l$ of sample $c_{1}$ given a data point in interval $k$ of column $c_{2}$.

Lemma 2. Conditional Entropy Matrix. Since $H\left(c_{j} \mid c_{i}\right) \neq H\left(c_{i} \mid c_{j}\right)$, the measure of dependence between $c_{i}$ and $c_{j}$ is represented in symmetric matrix form $\left(M_{c}\right)$ as:

$$
M_{c}\left(c_{i}, c_{j}\right)=\left\{\begin{array}{ll}
H\left(c_{i} \mid c_{j}\right) \times H\left(c_{j} \mid c_{i}\right) & \forall c_{i}, c_{j} \in C \quad \text { if } i \neq j \\
0 & \text { else }
\end{array}\right\} .
$$

\subsection{Construction of Unweighted Graph}

In order to map the generated symmetric matrix to the unweighted graph, we transformed the matrix by replacing all values greater than the entropy threshold to 0 and those less than the threshold to 1 . We consider graph $G(V, E)$ with node $V$ as a set of conditions and the edge $E$ as described below:

$$
E=\left\{\begin{array}{l}
\left.E_{i j}=1 \text { if } M_{c}\left(c_{i}, c_{j}\right) \leq \text { entropythreshold }\right) \\
E_{i j}=0 \text { else }
\end{array}\right\} .
$$

$E_{i j}=1$ denotes the cluster relationship between conditions $c_{i}$ and $c_{j}$ due to conditional entropy between two conditions lower than threshold $(\lambda)$. Moreover, $E_{i j}=0$ if there is no edge. We estimate the entropy threshold $(\lambda)$ as:

$$
\text { Entropy threshold }(\lambda)=M_{c}^{\min }+\beta\left(M_{c}^{\max }-M_{c}^{\min }\right),
$$

where $M_{\mathrm{c}}^{\min }$ and $M_{\mathrm{c}}^{\max }$ are minimum and maximum values in the conditional matrix $M_{c}$, respectively, and $\beta$ is an entropy ratio that has value ranges from 0.0 to 1.0.

\subsection{Finding the Cliques}

We enumerate the maximal cliques in the graph to identify groups of experimental conditions where relevant biclusters can be located. We utilize the Bron-Kerbosch maximum clique algorithm described in [13]. The algorithm operates by means of a backtracking tree search. It maintains three disjoint sets of nodes $R, P, X . R$ represents the currently growing clique; $P$ represents the prospective nodes which are connected to all nodes in $R . X$ contains nodes already processed, i.e. nodes which were previously in $P$. Hence, all maximal cliques have already been reported. All nodes which are connected to every node of $R$ are either in $P$ or $X$. The purpose of this work is to present a framework into which any effective clique enumeration algorithm can be plugged. 


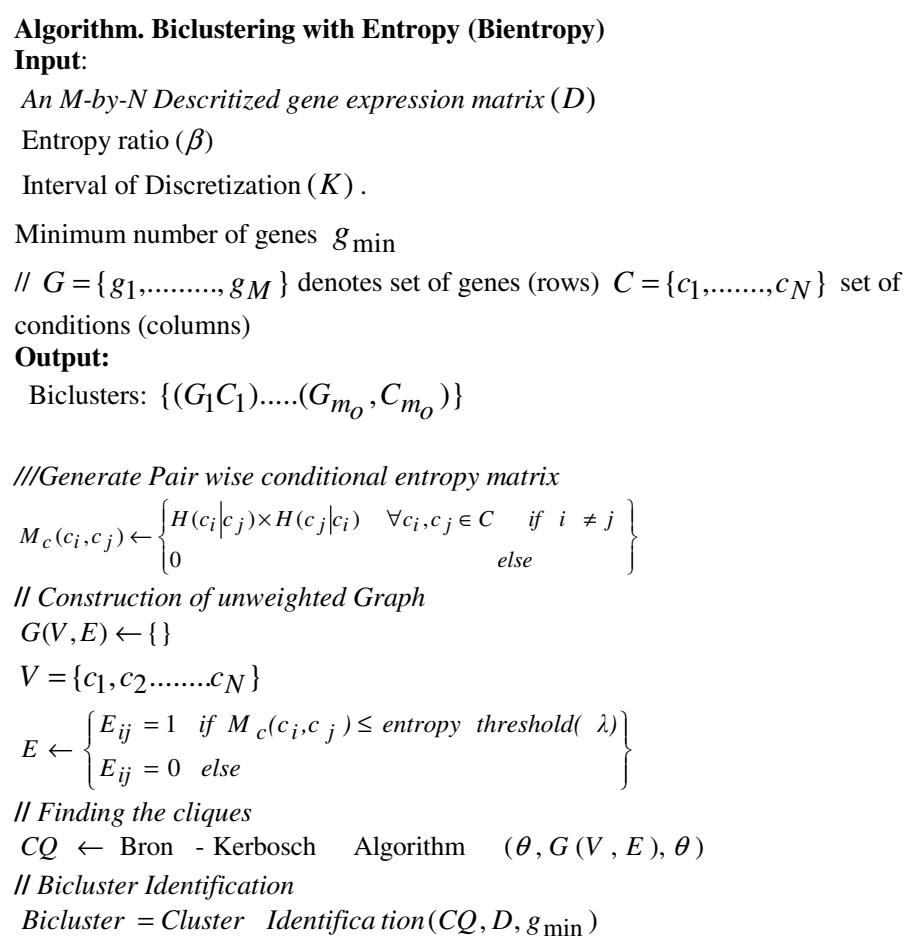

\subsection{Identification of Biclusters}

After identifying a group of correlated conditions through clique enumeration, we perform bicluster identification by identifying a group of genes correlated across the condition using conditional entropy, as described below.

$$
H\left(g_{1} \mid g_{2}\right)=-\sum_{k=1}^{K} P\left(g_{1}^{k}\right) \sum_{l=1}^{K} P\left(g_{1}^{l} \mid g_{2}^{k}\right) \log P\left(g_{2}^{l} \mid g_{1}^{k}\right) .
$$

Where $P\left(g_{1}^{k}\right)$ is the probability of data in discretization level $k$ of gene $g_{1}$, and $P\left(g_{1}^{l} \mid g_{2}^{k}\right)$ is the conditional probability of the data point in the interval $l$ gene $g_{1}$ given a data point in interval $k$ of gene $g_{2}$. The two genes are said to be strongly dependent if $H\left(g_{1} \mid g_{2}\right)=H\left(g_{1} \mid g_{2}\right)=0$.

We identify biclusters for each group of related conditions through the following procedure:

1. Generate a sub-matrix $M^{\prime}$ from existing discretized data, so that it has values of all genes that make up a clique,

2. Iteratively perform the following sub-steps until all the genes have been clustered, 
a. Randomly select a gene $g_{i}$ from $M^{\prime}$, and estimate the conditional entropy $g_{i}$ and each of the genes in $M^{\prime}$,

b. Identify genes with the conditional entropy equal to zero with gene $g_{i}$ and assign them to a cluster.

3. Remove identified genes from $M^{\prime}$.

\section{Complexity Analysis}

In the first stage, normalized expression levels of each gene are discretized into linear intervals $M$ and $N, O(M N)$. In the second stage, the conditional entropy matrix, which serves as an input to the clique graph is computed. We estimate conditional entropy among all pairs of conditions (samples) and represent the relationship as a graph. We have potential worst-case complexity of $O\left(N^{2}\right)$. In the third stage, we find the maximal cliques. If the number of cliques is small or the cliques are relatively small and disjointed, this operation is linear in $N$. In most cases, it will not significantly affect the overall efficiency by exceeding $O\left(N^{2}\right)$. Having found the cliques, stage four, involves the identification of biclusters through linear or log-linear experimental conditions $N$ and intervals $K$. Overall, assuming the maximal clique enumeration does not hit a 'hard' graph, the efficiency of the whole algorithm is $O(N(M+N+K))$.

\section{Implementation}

We implemented BiEntropy using MATLAB and input the normalized gene expression matrix and two parameters: $K$ and $\beta$, where $K$ is the number of discretization intervals and $\beta$ is an entropy ratio that ranges from 0 to 1 . We can choose $K=\{3,5,7,9\}$ for the discretization level.

\section{Experimental Results}

Our objective is to show that BiEntropy discovers both constant and coherent (additive) biclusters with respect to noise level and overlap among biclusters when compared with other biclustering algorithms. In addition, we intend to demonstrate our algorithm's ability to find biclusters with biological significance in gene expression data. Other algorithms include CC [2], SAMBA [3], ISA [5], and Bimax [7]. Biclustering analysis tools (BICAT) developed by Prelic et al. [10] were used to implement Bimax, ISA, CC, OPSM [4], and xMotif [6]. EXPANDER software developed by Maron-Katz et al. [11] was also used to implement SAMBA. The parameters of these algorithms were set to the values recommended in the corresponding publications. 


\subsection{Synthetic Dataset}

Our model for the generation of synthetic gene expression data is based on the proposal from Zimmerman et al. [7]. This dataset includes data matrices with three types of artificial implanted modules: constant, coherent (additive), and overlapping. For the constant situation, the matrix with implanted constant bicluster is generated in four steps. (1) Generate a 100*100-background matrix $A$ so that all elements of A are 0 . (2) Generate ten biclusters of size $10 * 10$, so that all elements of the biclusters are 1 . (3) Add noise to the biclusters by adding random values from uniform distribution $(-\sigma, \sigma)$. (4) Implant 10-biclusters to $A$ without overlap. We define the noise level from 0.0 to 0.25 for all experimentation. Ten modules (biclusters) are implanted to background matrix $A$. We use ten degrees of overlap $(d=0,1 \ldots \ldots \ldots . .9)$, where the size of the background matrix and modules vary from $100 \times 100$ to $110 \times 110$ and from $10 \times 10$ to $20 \times 20$, respectively. In coherent (additive) data, the procedure is the same as that of the constant data type, but we let the biclusters have a 0.02 increasing trend on the rows and columns.

To validate the accuracy of our algorithm, we apply the gene match score proposed by Prelic et al. [5]. Let $M_{1}$ and $M_{2}$ be two sets of biclusters. The match score of $M_{1}$ with respect to $M_{2}$ is:

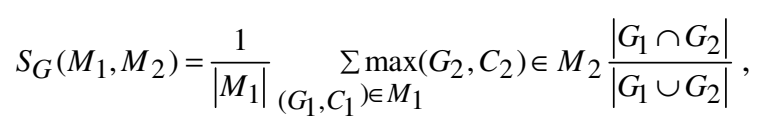

where $G$ and $C$ are a set of genes and a set of conditions in a bicluster, respectively. This score measures the degree of similarity between the computed biclusters and the true transcribed modules implanted in the synthetic data. Let $M_{\text {opt }}$ be the set of implanted modules, and let $M$ be the set of biclusters obtained by a biclustering algorithm. The average relevance, $S\left(M, M_{\text {opt }}\right)$, represents the extent to which the generated biclusters match true modules in the gene dimension. In contrast, the average module recovery, given by $S\left(M_{o p t}, M\right)$ quantifies how well each true bicluster is recovered by the biclustering algorithm under consideration. Both scores take the maximum value of 1 if $M_{\text {opt }}=M$.

\subsection{Parameter Selection}

The two parameters needed to implement our algorithm are discretization interval $(K)$ and Entropy ratio $(\beta)$. Since the entropy threshold depends on the entropy ratio values, we implement our biclustering method using entropy ratio values between 0 and 1 . We use a synthetic dataset with $100 * 100$ matrix and implant 10 non-overlapping $10 * 10$ constant biclusters. Figure 2 shows the high performance of the algorithms at entropy ratio value between 7.5 and 9.0 on three discretization intervals. Out of three discretization interval trials, 5-interval discretization gives the best average match score in our implementation. 


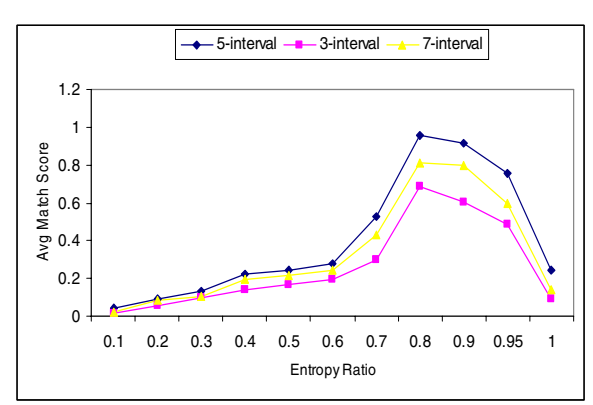

Fig. 2. Effect of Entropy ratio significantly enriched by GO

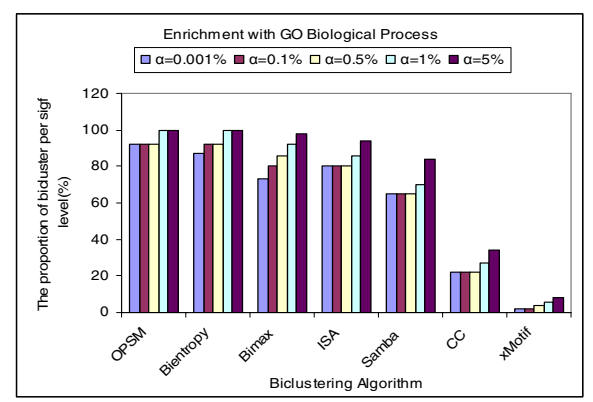

Fig. 3. The proportion of bicluster

\subsection{Effect of Noise}

To show the performance of BiEntropy against noise, we summarize the results in both constant and additive data in Figures $4 \mathrm{a}$ and $4 \mathrm{~b}$. In constant bicluster, BiEntropy, ISA, and Bimax show a high accuracy in the absence of noise, but the relevance and recovery scores obtained by $\mathrm{CC}$ and xMotif are statistically low. This phenomenon occurs because the algorithms do not focus on changes in gene expression but consider the similarity of the selection the only clustering criteria. When the noise level is high, ISA and BiEntropy have the best accuracies. The performance of the algorithms in Figure 4b shows that only three algorithms (BiEntropy, ISA, and SAMBA) demonstrate robustness against noise in additive biclusters. The figure also shows that

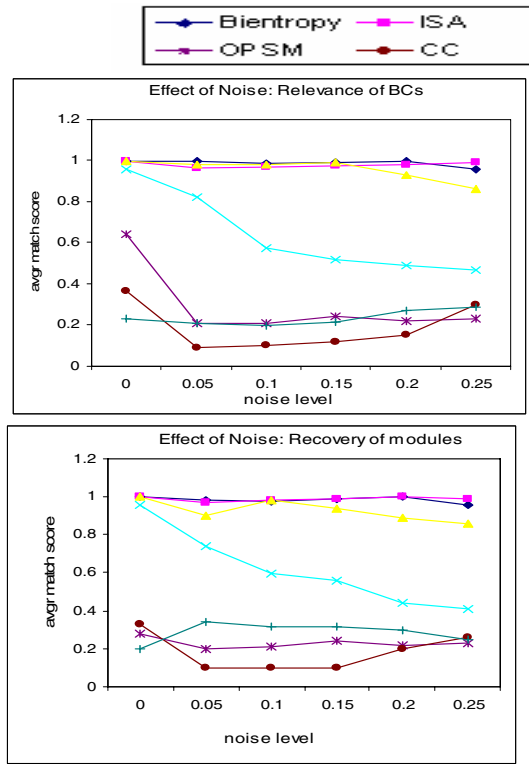

(a)
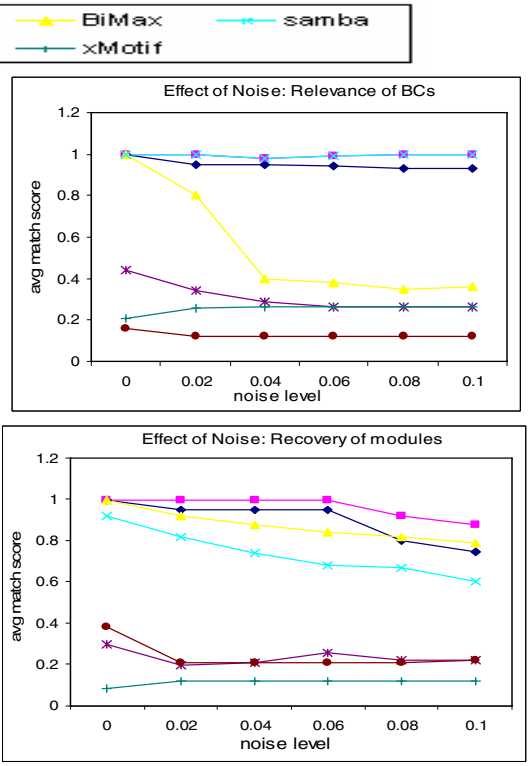

(b)

Fig. 4. Results of experiments on synthetic dataset: non-overlapping module with increasing noise level for (a) constant and (b) additive biclusters 
Bimax has a high accuracy with a constant bicluster but performs poorly in an additive data type at a high noise level.

\subsection{Effect of Overlapping Biclusters}

The goal of this section is to study the behavior of the chosen biclustering methods with respect to increased regulatory complexity. Figures $5 \mathrm{a}$ and $\mathrm{b}$ elaborate the performance of biclustering methods at different overlap degrees in the absence of noise. Bimax is the only method that fully recovers all hidden modules in the data matrix among the methods. BiEntropy and SAMBA also perform considerably well when compared to the remaining methods. OPSM is not significantly affected by the degree of overlap in additive bicluster, but it cannot handle constant bicluster with identical expression values.

ISA appears more sensitive to a high degree of overlap, especially with the additive biclusters. As with $\mathrm{CC}$, the performance increases with larger overlap degrees, but the gene match scores are still lower than those by Bimax, BiEntropy, SAMBA, and ISA, due to the diminishing number of background cells with large overlaps.

\subsection{Real Data}

We apply our biclustering method on the Saccharomyces cerevisiae dataset. The dataset, which has 2,993 genes and 173 conditions, is provided by Gasch et al. [8], and is available at http://www.tik.ee.ethz.ch/sop/bimax.We follow the approach used by Zimmerman et al. [7] to evaluate the performance of BiEntropy against other biclustering methods in respect to real expression data. A web tool called FuncAssociate [9] was used to evaluate discovered biclusters using Gene Ontology (GO) annotations [12]. Table 1 lists the parameter settings and the number of biclusters identified from each method. We filter out both biclusters with more than $25 \%$ overlap with large biclusters and output the rest in order of descending size. The adjusted significance score of each discovered bicluster is computed from FuncAssociate. The histogram in Figure 3 shows the proportion of biclusters from each method that contains one or several overrepresented GO categories for the Saccharomyces cerevisiae dataset. Bientropy and OPSM obtain the best results.

We attribute the good performance of BiEntropy to its unbiased discretization scheme, which accurately classifies bicluster types enriched with the GO Biological Process. OPSM performs well because it returns a small number of biclusters. Bimax, ISA, and SAMBA also provide a high portion of functionally enriched biclusters. Bimax and ISA ( $~ 90 \%$ at a significance level of $5 \%)$, have a slight advantage over SAMBA $(\sim 80 \%$ at a significance level of $5 \%)$. In contrast, CC and xMotif perform poorly. The scores for CC are $\sim 30 \%$.

Dataset NCi60 represents the gene expression patterns of 9703 genes in 60 human cancer cell lines and is available at http://www.discover.nci.nih.gov/nature2000. The complete data set contains missing values. We first select genes that have, at most, three missing values. There are 8161 such genes. We use the k-nearest neighbors impute function in MATLAB to estimate the missing values. We then calculate the variance for each gene expression profile and filter out the expression profiles with a variance of less than the $25^{\text {th }}$ percentile of the whole expression. The total number of genes left after 
filtering is 6344. We apply BiEntropy on the $\mathrm{NCi60}$ dataset with parameters $K=5$, and $\beta=0.9$ to generate 92 biclusters, 76 of which are selected after filtering out those with more than $25 \%$ overlap with larger biclusters. We evaluate the discovered biclusters by calculating the hyper-geometric functional enrichment score using FuncAssociate. Table 2 shows the partial results of the biclusters found by BiEntropy.

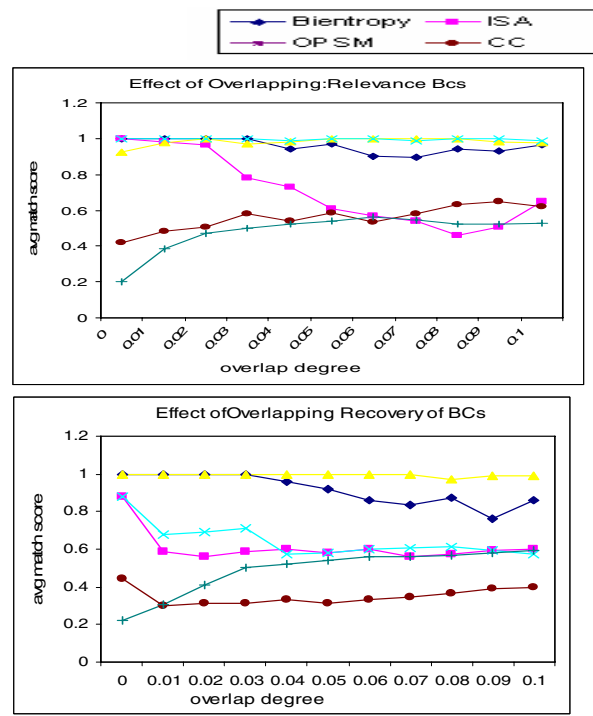

(a)
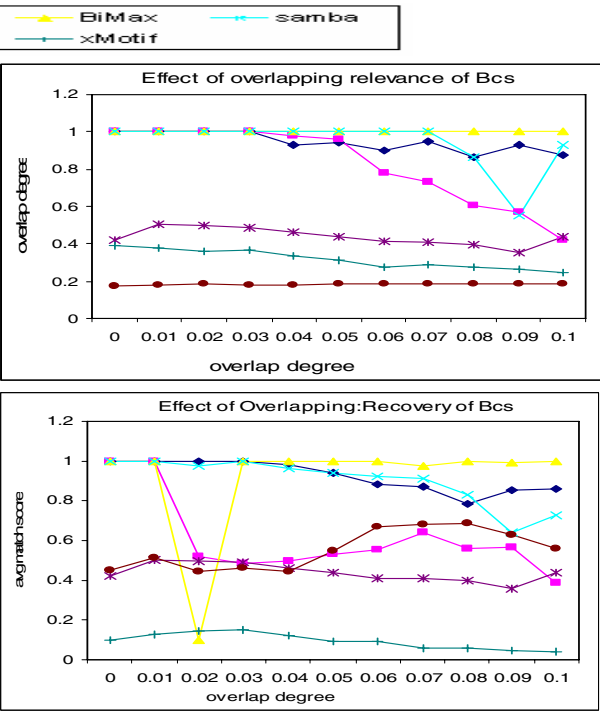

(b)

Fig. 5. Results of experiments on synthetic dataset: overlapping module with increasing overlap degree and noise for (a) constant and (b) additive biclusters

Table 1. Summary of parameters settings and total number of biclusters

\begin{tabular}{|l|l|l|l|}
\hline Algorithm & Default Parameters Setting & Values Used & $\begin{array}{l}\text { Total } \\
\text { Biclusters }\end{array}$ \\
\hline Samba & $D=40, N_{1}, N_{2}=6, k=20, L=30$ & default & 100 \\
\hline ISA & $\begin{array}{l}t_{g}=1.8 \sim 4.0(\text { step } 0.1), t_{c}=2.0, \\
n r \text { seeds }=20000\end{array}$ & $t_{g}=2.0$, sedds $=500$ & 66 \\
\hline OPSM & $l=100$ & default & 12 \\
\hline ZMotifs & $\begin{array}{l}n_{s}=10, n_{d}=1000, s_{d}=7 \sim 10 \\
\alpha \text { is not given, } p \text { - value }=10^{-40}, \text { max } \\
\text { length not given }\end{array}$ & $\begin{array}{l}s_{d}=7, \alpha=0.1, \\
\text { max_length }=0.7 m\end{array}$ & 306 \\
\hline CC & $\begin{array}{l}\alpha=1.2, \delta=\text { lower end of the exp ression } \\
\text { valuerange }\end{array}$ & $\delta \leq 0.5$ & 100 \\
\hline Bimax & $\begin{array}{l}\text { Min no. of genes not available } \\
\text { Min no. of chips not given }\end{array}$ & $\begin{array}{l}\text { min_genes }=12, \\
\text { min_chip } s=11\end{array}$ & 100 \\
\hline Bientropy & $\beta=0.0 \sim 1.0$, & $\beta=0.9$ & 74 \\
\hline
\end{tabular}

Table 2. Partial results of bicluster found in NCI60; size is given by the \# of gene

\begin{tabular}{|c|c|c|c|}
\hline \begin{tabular}{|l|} 
Bicluster \\
\end{tabular} & Sire & P-value & Aurotation \\
\hline Bicluster 1 & 86 & $1.80 \mathrm{E}-14$ & RHA metabolism \\
\hline Bicluster 23 & 82 & $2.60 \mathrm{E}-11$ & Fhysiological process \\
\hline \begin{tabular}{|l|} 
Bicluster 21 \\
\end{tabular} & 71 & $800 \mathrm{E}-9$ & $\begin{array}{l}\text { Cytoplastn organization and } \\
\text { biogenesis }\end{array}$ \\
\hline Bicluster 14 & 68 & $5.40 \mathrm{E}-7$ & BidingLigand \\
\hline Bicluster 29 & 63 & $5.70 \mathrm{E}-6$ & Protein Cotuplex \\
\hline \begin{tabular}{|l|} 
Bicluster 41 \\
\end{tabular} & 54 & $4.60 \mathrm{E}-6$ & Ribosottle \\
\hline Bicluster 32 & 39 & $5.30 \mathrm{E}-5$ & Cellular biosynthesis \\
\hline \begin{tabular}{|l} 
Biclustef 7 \\
\end{tabular} & 28 & $390 \mathrm{E}-5$ & metal binding \\
\hline Bicluster 39 & 19 & $1.50 \mathrm{E}-5$ & $\begin{array}{l}\text { Receptof Signalitig P totein } \\
\text { activity }\end{array}$ \\
\hline Bicluster 14 & 17 & $2.50 \mathrm{E}-3$ & Respontse to Stitululus \\
\hline
\end{tabular}




\section{Conclusion}

We have proposed and implemented a novel biclustering method, called BiEntropy, to discover constant, coherent (additive), and overlapped biclusters in the presence of noise. The method combines conditional entropy and graph theoretic techniques to identify a subset of conditions in which biclusters can be located. The experimental results on both synthetic and real data (Saccharomyces cerevisiae and NCI 60) reveal that BiEntropy is robust against noise and overlaps in both constant and additive biclusters. Our evaluation framework also shows a better accuracy than most biclustering methods.

\section{References}

[1] Hartigan, J.: Direct Clustering of a Data Matrix. J. Am. Statistical Assoc. 67, 123-129 (1972)

[2] Cheng, Y., Church, G.M.: Biclustering of Expression Data. In: Proceedings of Intelligent Systems for Molecular Biology (2000)

[3] Kupiec, M., Shamir, R., Tanay, A., Sharan, R.: Revealing Modularity and Organization in the Yeast Molecular Network by Integrated Analysis of Highly Heterogeneous GenomeWide Data. PNAS 101, 2981-2986 (2004)

[4] Karp, R., Ben-Dor, A., Chor, B., Yakhini, Z.: Discovering Local Structure in Gene Expression Data: The Order-Preserving Sub Matrix Problem. In: Proceedings of the 6th Int. Conf. on Computational Molecular Biology (RECOMB), pp. 49-57 (2002)

[5] Bergmann, S., Ihmels, J., Barkai, N.: Defining Transcription Modules Using Large-Scale Gene Expression Data. Bioinformatics 20, 2003-2004 (1993)

[6] Murali, T.M., Kasif, S.: Extracting Conserved Gene Expression Motifs from Gene Expression Data. In: Proceedings of the 8th Pacific Symposium on Biocomputing

[7] Zimmermann, P., Wille, A., Buhlmann, P., Gruissem, W., Hennig, L., Thiele, L., Zitzler, E., Prelic, A., Bleuler, S.: A Systematic Comparison and Evaluation of Biclustering Methods for Gene Expression Data. Bioinformatics (2006)

[8] Gasch, A.P.: Genomic Expression Programs in the Response of Yeast Cells to Environmental Changes. Mol. Biol. Cell 11, 4241-4257 (2000)

[9] Berriz, G., Bryant, O., Sander, C., Roth, F.: Charactering Gene Sets with FuncAssociate. Bioinformatics 22, 1282-1283 (2003)

[10] Prelic, A., Zimmermann, P., Barkow, S., Bleuler, S., Zitzler, E.: Bicat: A Biclustering Analysis Toolbox. Bioinformatics 22, 1282-1283 (2006)

[11] Maron-Katz, A., Sharan, R., Shamir, R.: Click and Expander: A System for Clustering and Visualizing Gene Expression Data. Bioinformatics 19, 1787-1799 (2003)

[12] GeneOntology Consortium, http: / / www . geneontology . org

[13] Bron, C., Kerbosch, J.: Algorithm 457: Finding All Cliques of an Undirected Graph. ACM Comm. 16 (1973) 